УДК 617.51/.53-006.6-085.849.2-06-08-039.71:612.017.1 doi:10.25298/2221-8785-2021-19-2-212-218 ИЗМЕНЕНИЕ ПОКАЗАТЕЛЕЙ ИММУНИТЕТА ПРИ ЛУЧЕВОЙ ТЕРАПИИ РАКА ГОЛОВЫ И ШЕИ В ЗАВИСИМОСТИ ОТ МЕТОДА ПРОФИЛАКТИКИ ЛУЧЕВЫХ РЕАКЦИЙ

\author{
Л. Б. Пархоменко
}

Белорусская медицинская академия последипломного образования, Минск, Беларусь

Введение. Применение магнитолазера и локальной гипоксии во время лучевой терапии рака головы и шеи приводит к снижению острой токсичности.

Цель. Снизить выраженность лучевых реакций у пащฺиентов, страдающих раком головы и шеи, с помощъью разработанных методов профилактики.

Материал и методы. В проспективное рандомизированное исследование вошли 120 пациентов, страдающих раком головы и шеи. Проводилась сравнительная оценка эффективности трех разработанных методов профилактики по сравнению с традиционной профилактикой.

Результаты. Использование магнитолазера, локальной гипоксии и их комбинации привело к снижению уровня провоспалительных цитокинов и увеличению концентрации противовоспалительных циитокинов по сравнению с контролем. Это соответствовало меньшей степени лучевых реакиий в основных группах.

Bыводы. Применение магнитолазера и/или гипоксии снижает острую токсичность, что подтверждается изменениями показателей иммунитета.

Ключевые слова: рак органов головы и шеи, лучевые реакции, магнитолазер, локальная гипоксия, ичтокиньл.

Для цитирования: Пархоменко, Л. Б. Изменение показателей иммунитета при лучевой терапии рака головь и шеи в зависимости от метода профилактики лучевых реакций / Л. Б. Пархоменко // Журнал Гродненского государственного медицинского университета. 2021. T. 19, № 2. C. 212-218. https://doi.org/10.25298/2221-8785-2021-19-2-212-218.

\section{введение}

Лучевая терапия плоскоклеточного рака органов головы и шеи сопровождается развитием ранних лучевых реакций слизистой оболочки полости рта и кожи. Частое и тяжелое раннее осложнение лучевой терапии - мукозит III-IV степени $[1,2]$. Для профилактики его развития или лечения традиционно используются обезболивающие, противовоспалительные и антимикробные препараты, антиоксиданты и витамины, местное охлаждение тканей полости рта, низкоинтенсивное лазерное излучение $[3,4,5$, 6]. Перспективным считается одновременное использование лазерного излучения и магнитных полей [7]. Однако до сих пор не существует общепринятых высокоэффективных протоколов профилактики и лечения.

Для снижения частоты и выраженности ранних лучевых реакций и осложнений в нашем исследовании были изучены результаты применения магнитолазерной терапии и локальной гипоксии в процессе лучевой терапии у пациентов со злокачественными новообразованиями органов головы и шеи с динамикой уровня концентрации цитокинов.

На современном этапе наиболее изученными цитокинами, которые появляются в процессе развития иммунного ответа и служат медиаторами иммунной и воспалительной реакций, являются цитокины иммунной системы и факторы роста. По своему действию цитокины иммунной системы делятся на провоспалительные и противовоспалительные. Провоспалительные цитокины (интерлейкины IL-1, IL-2, IL-6, хемокин IL-8, фактор некроза опухоли TNF- $\alpha$, интерфероны IFN- $\alpha$, IFN- $\beta$, IFN- $\gamma$ и другие) действуют на иммунокомпетентные клетки, иници- ируя воспалительный ответ. Высокий уровень этих цитокинов может говорить об активности и тяжелой степени патологического процесса. Противовоспалительные цитокины (IL-4, IL10, IL-13 и другие) регулируют специфические иммунные реакции, ограничивая развитие воспаления. Кроме того, существуют регуляторы клеточного и гуморального иммунитета, обладающие собственными эффекторными цитотоксическими функциями. Из CD4+ Т-клеток образуются субпопуляции Т-хелперов 1 типа (Th1) и Т-хелперов 2 типа (Th2). Участвуя в процессе клеточного иммунитета, Th1 активируют макрофаги, продуцируя IL-2 и IL-3 и INF- $\gamma$. Th2 вызывают синтез антител B-клетками, секретируя IL4, IL-10, IL-13. Эти цитокины, продуцируемые разными субпопуляциями Т-хелперов, обладают взаимоингибирующим действием. Например, INF- $\gamma$ подавляет пролиферацию Th2, a IL-10 Th1. Характер ответа, соответствующий Th1 или $\mathrm{Th} 2$, зависит от взаимодействия или с макрофагами, секретирующими IL-12, или с Т-клетками, продуцирующими IL-4. Нарушение баланса активности Th1 и Th2 играет большую роль в хронизации и прогрессировании воспалительного процесса $[8,9,10]$.

Цель исследования - снизить выраженность лучевых реакций у пациентов, страдающих раком головы и шеи, с помощью разработанных методов профилактики.

\section{Материал и методы}

Дизайн исследования. На базе ГУ «РНПЦ онкологии и медицинской радиологии им. Н. Н. Александрова» в 2010-2012 гг. проведено проспективное рандомизированное исследование по применению магнитолазерной и гипоксической терапии для профилактики раз- 
вития тяжелых лучевых реакций у пациентов, страдающих раком головы и шеи.

Критерии включения пациентов в исследование: морфологическая верификация плоскоклеточного рака полости рта, языка, глотки и гортани II, III или IVA, В стадий; возраст от 18 до 70 лет; наличие измеряемой опухоли; показатель функционального состояния по шкале Карновского более 70\% (0-1 балл по шкале ECOG); отсутствие сопутствующей патологии в стадии декомпенсации, отсутствие в анамнезе облучения области головы и шеи и/или курсов химиотерапии по поводу другого злокачественного новообразования. Все пациенты подписали информированное согласие на участие в исследовании.

Пациенты. В исследование были включены 180 пациентов. До начала рандомизации у 60 пациентов, не включенных в заключительный анализ, проводилась отработка методов магнитолазерной и гипоксической терапии в разных режимах. После установления отсутствия каких-либо побочных эффектов применения магнитолазеротерапии и фармакологически индуцированной локальной гипоксии во время облучения были сформированы группы для сравнительного изучения эффективности ранней медицинской реабилитации, включающей стандартные подходы и новые методы профилактики и лечения ранних лучевых реакций. Рандомизацию прошли 120 пациентов в возрасте 32-76 лет. Двадцать два пациента выбыли из протокола по техническим причинам или после отказа от дальнейшего участия в клиническом исследовании, или из-за обострения сопутствующей патологии.

После рандомизации в группу «Контроль» вошли 32 пациента с диагнозом рака дна полости рта или языка (6 пациентов), ротоглотки (14), гортаноглотки (6) и гортани (6). В группу «Магнитолазер» вошли 20 пациентов с диагнозом рак полости рта или языка (5 пациентов), ротоглотки (6), гортаноглотки (2), гортани (7). В группу «Гипоксия» вошли 25 пациентов с диагнозом рак дна полости рта или языка (6 пациентов), ротоглотки (13), гортаноглотки (5) и гортани (1). В группу «Комплекс» вошел 21 пациент с диагнозом рак дна полости рта или языка (2 пациента), ротоглотки (13), гортаноглотки (2) и гортани (4).

Всем пациентам проводилась дистанционная 3D-конформная конвенциональная лучевая терапия РОД 2 Гр, 1 раз в день, 5 раз в неделю, до СОД 66-70 Гр на опухоль и СОД 50-70 Гр на регионарные лимфатические узлы шеи.

Одновременную химиолучевую терапию с внутривенным введением цисплатина $100 \mathrm{mг} / \mathrm{M}^{2}$ в первый, 22 и 43 дни облучения получали 70\% пациентов, включенных в исследование.

При сравнении групп исследования по возрасту и степени распространенности опухолевого процесса не выявлено статистически значимых различий.

Методики профилактики и лечения ранних лучевых реакичй. В группе «Контроль» во время курса лучевой терапии проводилась санация полости рта антисептическими растворами 8-10 раз в день (отвар шалфея, ромашки и др.). При болевом синдроме местно осуществлялись аппликации 1-2\% раствором лидокаина, анестезина, новокаина.

В группе «Магнитолазер» с третьего дня облучения через день назначалась магнитолазерная терапия в течение 5-7 минут с воздействием на слизистую оболочку полости рта и ротоглотки, кожные покровы сканирующим методом с зазором между излучателем и облучаемыми тканями 0,5-1,0 см через 60 минут после сеанса лучевой терапии. Лазерное излучение с частотой воздействия лазера 1000 Гц и импульсной мощностью лазера 8 Вт; постоянное магнитное поле 30 млТл.

В группе «Гипоксия» использовалась местная гипоксия путем орошения визуально неизмененной слизистой оболочки полости рта спреем ксилометазолина гидрохлорида 0,1\% (максимальная доза - до 4 впрыскиваний).

В группе «Комплекс» осуществлялась комплексная терапия, включающая гипокситерапию до сеанса облучения и магнитолазерную терапию после сеанса лучевой терапии по приведенным выше методикам.

Конечные точки исследования. В качестве конечных точек использовались показатели острой токсичности слизистой оболочки и кожи. Выраженность ранних лучевых реакций нормальных тканей оценивали по шкале EORTC/RTOG. BToричные конечные точки включали изменение показателей клеточного и гуморального иммунитета. Вместе с клинической оценкой изменений нормальных тканей изучались количественные изменения концентрации воспалительных и провоспалительных цитокинов в периферической крови пациентов перед началом, в середине и в конце курса лучевой терапии.

\section{Статистические методы}

Сравнение реабилитационных показателей в группах до и после лучевой терапии проводилось по критерию предельной однородности при повторяющихся измерениях. Сравнение групп по реабилитационным показателям проводилось по критерию хи-квадрат; в случае нарушения предположений, лежащих в основе критерия хи-квадрат, использовался критерий Фишера. Для последующих попарных сравнений применялась поправка Хольма на множественные сравнения для предотвращения ложноположительных результатов в рамках заданной ошибки первого рода. Для оценки взаимосвязи между степенью лучевых реакций слизистых оболочек и изменением количества цитокинов в крови пациентов использовался коэффициент корреляции Спирмена. Результаты считались статистически значимыми при $\mathrm{p}<0,05$.

\section{Результаты и обсуждение}

Безопасность. V степени лучевых реакций (смерть от лучевых осложнений) не зафиксировано ни в одной из групп исследования. Все пациенты, которым проводились разработанные реабилитационные мероприятия, завершили за- 
планированный курс радиотерапии без продолжительных перерывов в специальном лечении. Серьезных побочных реакций общего или местного характера ни в одной из групп пациентов не выявлено.

Результаты исследования клеточного и гуморального иммунитета. У пациентов, страдающих онкологическим заболеванием, нарушена функция клеточного звена иммунной системы, а гуморальное звено функционирует нормально. Таким образом, подавлена активность Т-хелперов 1 типа и сохранена активность Т-хелперов 2 типа. Основные нарушения отмечаются в Т-клеточном звене иммунитета, что связано с изменением соотношения иммунорегуляторных субпопуляций Т-лимфоцитов (CD4+/CD8+) и снижением функциональной активности и количества натуральных киллеров NK, активирующих процесс антиген-независимого лизиса клеток.

В нашем исследовании у пациентов с плоскоклеточным раком органов головы и шеи фенотипирование В-лимфоцитов (CD19+) периферической крови выявило снижение их процентного содержания в контрольной группе до 1,8. При использовании магнитолазера и/или гипоксии данный показатель был равен 5,2\% при его нормальном уровне $12-12,5 \%$ (рис. 1 ).

Снижение содержания Т-лимфоцитов (CD3+) установлено во всех группах: в контрольной группе - в 1,21 раза, в трех основных группах - в 1,01 раза.

Выявлено снижение содержания Т-хелперов $(\mathrm{CD} 3+\mathrm{CD} 4+)$ до $31 \%$ в контрольной группе и до $36 \%$ у пациентов в основных группах.

Содержание цитотоксических Т-лимфоцитов (CD3+CD8+) было увеличено у пациентов контрольной группы в $1,13 \%$ раза. В основных группах установлено незначительное снижение данного показателя до 23\% при нормальном показателе 26-28\%.

Итак, субпопуляционные сдвиги Т-лимфоцитов привели к дисбалансу иммунной системы с

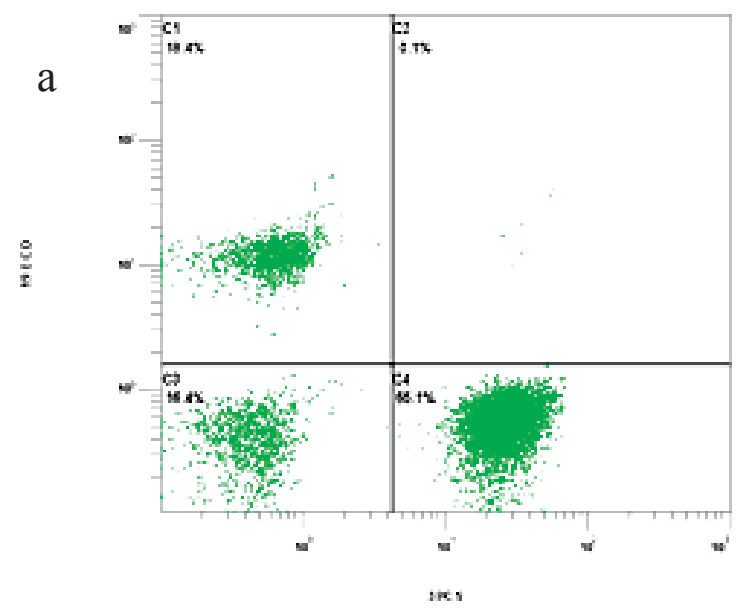

уменьшением иммунорегуляторного индекса CD4+/CD8+.

Снизилось содержание натуральных киллеров (CD16+CD56+) до 5,6\% (в 2,77 раза) в контрольной группе; до 8,5\% (в 1,83 раза) в исследуемых группах. Незначительно (до 19,9\%) уменьшилось содержание активационного антигена HLA-DR у пациентов с комплексным воздействием. В контрольной группе этот показатель находился в пределах нормы.

Концентрация иммуноглобулина G (IgG) в группах находилась в пределах от 12,613 до 18,771 мг/мл при нормальном значении 8,4-13,2 мг/мл. В группе «Магнитолазер» имелось постепенное восстановление его уровня: в начале курса ЛТ - 15,038 мг/мл, в середине - 14,648 мг/мл и в конце - 13,269 мг/мл. В группе «Комплекс» в начале облучения наблюдалась активация синтеза IgG, однако в середине и конце ЛТ его уровень снизился в 1,3 раза по отношению к первоначальным значениям (16,327 мг/мл - в начале, 12,613 мг/мл - в середине и 12,263 мг/мл - в конце терапии). В группе «Гипоксия» в динамике уровня концентрации $\operatorname{IgG}$ не выявлено статистически значимых различий.

Достоверное увеличение концентрации $\operatorname{IgA}$ установлено во всех анализируемых группах от 2,355 мг/мл до 4,510 мг/мл при нормальном значении 1,4-4,0 мг/мл. В группах «Комплекс» и «Гипоксия» отмечено снижение его уровня концентрации в динамике. Однако при гипоксии наблюдалось более низкое содержание IgA: 4,510 мг/мл и 2,954 мг/мл - в начале, 3,652 мг/ мл и 2,852 мг/мл - в середине, 3,534 мг/мл и 2,355 мг/мл - в конце лечения. В группе «Магнитолазер» зафиксировано увеличение его уровня в середине курса и стабилизация в конце: 3,088 мг/мл - в начале, 3,339 мг/мл - в середине, 2,968 мг/м - в конце облучения.

Концентрация IgM в группах исследования варьировала от 0,732 до 2,453 мг/мл при нормальном значении 0,5-1,8 мг/мл. В группе «Маг-

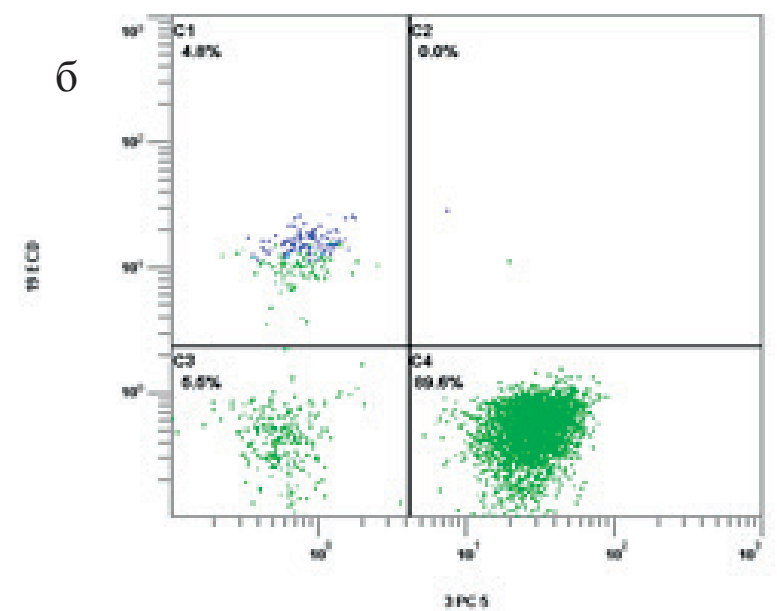

а) нормальное распределение; б) патологическое распределение

Рисунок 1. - Распределение лимфоцитов по параметрам флуоресценции при окраске антителами к СD19+ клеткам периферической крови пациентов

Figure 1. - Distribution of lymphocytes by fluorescence parameters when stained with antibodies to CD19 + peripheral blood cells of patients 
нитолазер» концентрация $\operatorname{IgM}$ увеличилась в середине терапии и имела тенденцию к снижению по отношению к первоначальным значениям: 1,626 мг/мл - среднее значение в начале, 1,921 мг/мл - в середине, 1,429 мг/мл - в конце облучения. В группе «Гипоксия» имелось снижение концентрации $\operatorname{IgM}$ в 1,9 и 1,5 раза в середине и конце курса: 2,453 мг/мл - в начале, 1,323 мг/мл - в середине, 1,612 мг/мл - в конце.

Результаты исследования провоспалительных и противовоспалительных цуитокинов. Одну из центральных ролей в воспалительной реакции играет интерлейкин-1 (IL-1), являясь стимулятором ее развития. В нашем исследовании выявлено стойкое снижение его концентрации в крови пациентов группы «Магнитолазер»: 14,457 пг/ мкл - среднее значение концентрации в начале, 13,583 пг/мкл - в середине и 12,042 пг/мк - в конце лечения (рис. 2).

У пациентов группы «Гипоксия» во время курса облучения отмечено увеличение концентрации IL-1: в начале - 14,126 пг/мкл, в середине - 15,238 пг/мкл, в конце - 13,277 пг/ мкл. В группе «Комплекс» наблюдалось снижение уровня IL-1 в 1,24 раза в середине курса и незначительное увеличение по окончании 13,503 пг/мкл, 10,857 пг/мкл и 12,291 пг/мкл, соответственно. Концентрация IL-1 в группе «Контроль» имела тенденцию к неуклонному росту от 12,644 пг/мкл в начале до 12,488 пг/мкл в середине и 16,833 пг/мкл в конце лечения (возрастание концентрации в 1,33 раза).

Развитие воспалительной реакции сопровождается секрецией цитокинов «первого поколения», таких как IL-1 и IL-6, TNF- $\alpha$, которые индуцируют синтез центрального регуляторного цитокина IL-2, а также цитокинов «второго поколения» IL-3, IL-4, IL-5, IFN- $\gamma$. Они в свою очередь влияют на синтез ранних цитокинов.

Изучение концентрации IL-6 в периферической крови выявило наиболее эффективный метод профилактики острой токсичности - магнитолазерное воздействие, при котором этот показатель уменьшился в 2,25 раза к концу курса терапии: 19,238 пг/мкл - в начале, 11,014 пг/мкл - в середине и 8,543 пг/мкл - в конце лучевого лечения. В группе «Контроль» установлено увеличение (в 1,5 раза) концентрации IL-6 к концу облучения. Комплексное воздействие и местная гипоксия не повлияли на уровень IL-6 в периферической крови пациентов в процессе лечения.

Интерлейкин-8 - провоспалительный цитокин и фактор активации нейтрофилов. Он активирует нейтрофилы, в меньшей степени - другие гранулоцитарные лейкоциты, вызывая их хемотаксис к очагу воспаления. Повышенный уровень IL-8 связан с хроническим и острым воспалением, нейтрофильной инфильтрацией в тканях. Анализ изменений концентрации IL-8 в процессе лучевой терапии показал, что наиболее значимым противовоспалительным эффектом обладает магнитолазерная терапия. Уровень IL-8 в этой группе снизился в 1,3 раза в середине и в 1,6 раза в конце облучения по сравнению с первоначальным значением. В группе «Гипоксия» содержание IL-8 имело скачкообразный характер, снизившись к середине лечения в 1,1 раза и увеличившись к окончанию курса в 1,1 раза. В группах «Контроль» и «Комплекс» концентрация IL-8 увеличилась по отношению к первоначальным значениям: соответственно, 14,345 пг/мкл и 12,953 пг/мкл - в начале, 20,315 пг/мкл и 19,315 пг/мкл - в середине, 18,372 пг/мкл и 15,771 пг/мкл - в конце терапии.

Важную роль в реализации механизмов иммунного ответа играет интерлейкин-2, который является центральной регуляторной молекулой, в том числе формирует эффекторный иммунологический ответ, направленный на предотвращение пролиферации неопластических клеток. У пациентов группы «Магнитолазер» выявлено снижение концентрации IL-2 в середине облу-

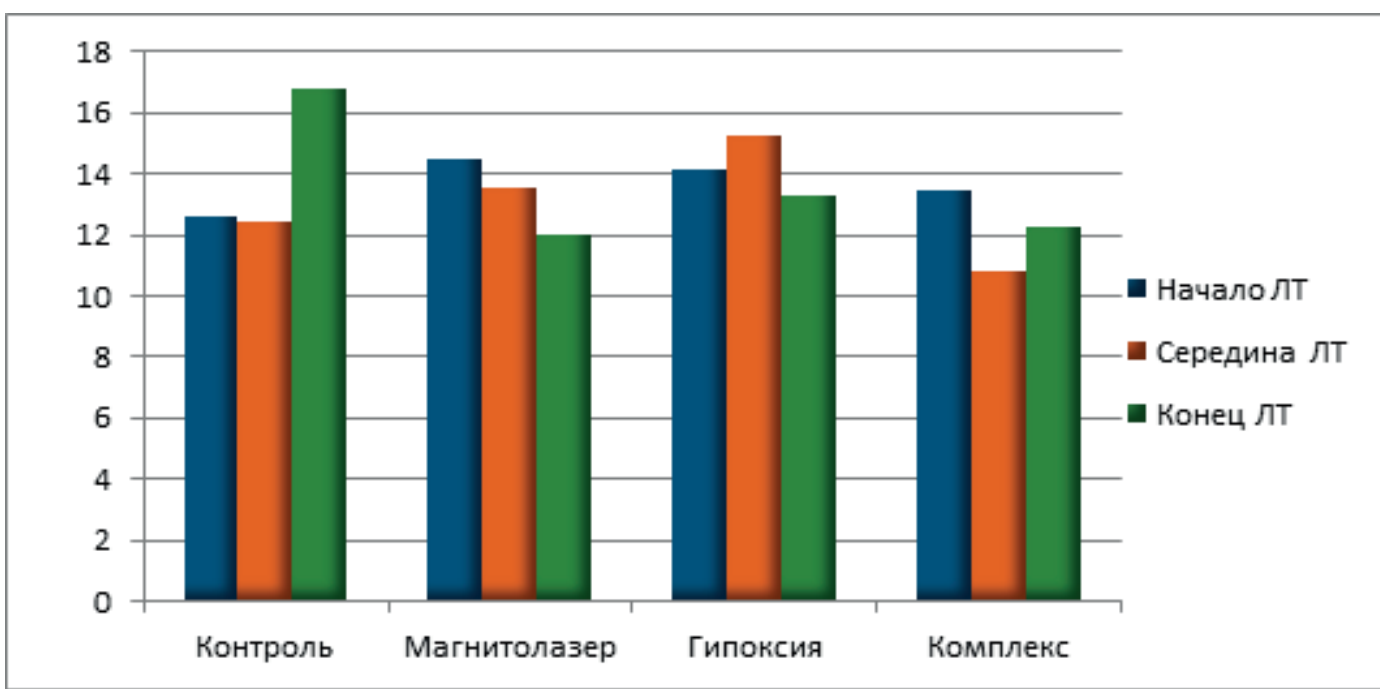

Рисунок 2. - Динамика концентрации IL-1 во время курса лучевой терапии в зависимости от метода профилактики

Figure 2. - Dynamics of IL-1 concentration during radiation therapy, depending on the method of prevention 
чения в 1,6 раза, но по окончании лечения его концентрация приближалась к первоначальным значениям. Это можно объяснить активацией иммунного процесса в начале лучевой терапии, частичным истощением резервов организма в середине лечения и нормализацией уровня в конце лечения $(15,293$ пг/мкл - в начале, 9,862 пг/мкл - в середине и 14,353 пг/мкл в конце терапии). У пациентов группы «Комплекс» выявлено снижение концентрации IL-2 к середине курса в 2,6 раза и увеличение его концентрации в конце курса, но не до первоначальных значений: 19,102 пг/мкл - в начале, 7,419 пг/мкл - в середине и 10,095 пг/мкл - в конце лучевой терапии. В группе «Гипоксия» на протяжении всего курса лечения концентрация IL-2 находилась примерно на одном уровне. В группе «Контроль» концентрация IL-2 уменьшилась к середине лечения и достигла первоначальных значений к концу облучения: 11,568 пг/мкл - в начале, 5,798 пг/мкл - в середине и 12,102 пг/мкл - в конце лучевой терапии.

Один из важных регуляторов дифференцировки Т- и В-лимфоцитов - интерлейкин-4. Он способен генерировать активность лимфокин-активированных клеток и усиливать противоопухолевую активность макрофагов. Оказывая сильный эффект на регуляцию образования других цитокинов, IL-4 в процессе реализации своей активности вначале значительно увеличивается и далее снижается для поддержания баланса и предотвращения хронизации процесса. В нашем исследовании установлено, что наибольшей противовоспалительной активностью обладает магнитолазерная терапия: среднее значение IL-4 в начале лучевой терапии составило 16,777 пг/мкл, в середине - 8,271 пг/мкл и в конце $-14,658$ пг/мкл.

В группе «Комплекс» наблюдалась активация синтеза IL-4 в начале лечения, снижение в 5,2 раза в середине и в 4,2 раза в конце облучения по отношению к первоначальным значениям (8,647 пг/мкл - в начале, 1,672 пг/мкл - в середине и 2,054 пг/мкл - в конце). В группе «Гипоксия» не выявлено статистически значимых различий концентрации IL-4 в процессе лечения. В группе «Контроль» к концу лучевой терапии произошло увеличение концентрации IL-4 до 16,214 пг/мкл.

Интерлейкин-10 продуцируется Т-хелперами 2 типа. Он служит одним из важных цитокинов-регуляторов воспаления, являясь мощным антагонистом некоторых цитокинов. IL-10 подавляет секрецию таких медиаторов воспаления, как IFN, TNF, IL-1 и IL-6. В нашем исследовании выявлено, что магнитолазер, гипоксия и их комплексное воздействие оказывают положительное влияние на продукцию IL-10 с тенденцией к его росту. В группе «Магнитолазер» концентрация IL-10 увеличилась в начале курса, снизилась на $31 \%$ в середине лечения и возросла в конце облучения $(8,345$ пг/мкл - в начале, 6,369 пг/мкл - в середине и 8,865 пг/мкл - в конце терапии).
В группе «Гипоксия» зафиксирован рост уровня концентрации IL-10 (5,56 пг/мкл - в начале, 6,185 пг/мкл - в середине и 7,554 пг/мкл - в конце). В группе «Комплекс» также отмечается увеличение его концентрации в процессе проводимого лечения $(7,328$ пг/мкл - среднее значение IL-10 в начале ЛТ, 7,403 пг/мкл - в середине и 9,067 пг/мкл - в конце). В группе «Контроль» концентрация IL-10 в конце лечения приближалась к значениям в трех основных группах. Средний уровень концентрации цитокина в контрольной группе составил 10,789 пг/мкл, что способствует постепенному истощению резервных возможностей организма и в конечном итоге приводит к дисбалансу про- и противовоспалительных цитокинов.

Интерлейкин-13 по своим биологическим функциям схож с IL-4. Он продуцируется активированными Т-лимфоцитами и является мощным модулятором активности моноцитов и В-клеток, оказывает ингибирующий эффект на продукцию цитокинов, стимулирующих начало воспалительного процесса. Его средняя концентрация у пациентов в группе «Магнитолазер» была повышена по сравнению с контрольной группой и находилась примерно на одном уровне: 12,955 пг/мкл - в начале, 13,221 пг/мкл - в середине и 13,825 пг/мкл - в конце облучения. В группе «Гипоксия» концентрация IL-13 увеличилась в 1,2 раза, затем приблизилась к первоначальным показателям (11,414 пг/мкл - в начале, 13,456 пг/мкл - в середине и 12,081 пг/мкл - в конце). В группе «Комплекс» установлено снижение уровня IL-13 (13,732 пг/мкл - в начале, 10,556 Пг/мкл - в середине и 11,211 пг/мкл - в конце терапии).

Биологические эффекты TNF- $\alpha$ заключаются в иммуномодулирующем действии, способности к геморрагическому некрозу опухоли. Многие эффекты связаны с активацией или ингибицией экспрессии определенных генов. TNF- $\alpha$ активирует транскрипцию генов других провоспалительных цитокинов. В группах «Магнитолазер» и «Комплекс» концентрация TNF- $\alpha$ имела тенденцию к снижению в процессе лечения: соответственно, 13,955 пг/мкл и 13,109 пг/мкл - в начале курса, 11,698 пг/мкл и 7,937 пг/мкл - в середине, 11,171 пг/мкл и 9,565 пг/мкл - в конце. В группе «Гипоксия» в ходе проведения лучевой терапии установлено увеличение концентрации TNF- $\alpha$, однако по отношению к остальным группам она была снижена в среднем в 1,5-2 раза (6,384 пг/мкл - в начале, 6,851 пг/мкл - в середине и 7,212 пг/мкл - в конце).

\section{Bыводы}

Анализ полученных данных показал, что магнитолазерное воздействие и сочетанное его использование с гипоксией для профилактики ранних лучевых реакций приводит к нормализации числа CD3+лимфоцитов, соотношения $\mathrm{CD}+4$ / CD8+ и концентрации сывороточных иммуноглобулинов к окончанию лечения. Иммунокорригирующий эффект комплексного воздействия достигается благодаря повышению содержания 
иммуномедиаторов. При этом происходит усиление продукции и увеличение количества циркулирующих иммуноглобулинов, повышение и восстановление уровня Т- и В-лимфоцитов, что оказывает существенный лимфостимулирующий эффект.

Выявлена корреляционная зависимость между степенью лучевых реакций слизистых оболочек и кожи и снижением уровня провоспалительных цитокинов $(\mathrm{R}=0,35, \mathrm{p}<0,05$, по Спирмену). Уменьшение концентрации провоспалительных цитокинов IL-1, IL-6 и IL-8 и TNF- $\alpha$ при разработанных методах профилактики соответствовало наименьшей степени лучевых реакций слизистых оболочек и кожи (0-1 степень), в то время как в контрольной группе наблюдалось увеличение концентрации данных показателей крови при более выраженной степени острой токсичности (2-3 степень).

Анализ данных выявил корреляционную взаимосвязь между степенью лучевых реакций слизистых оболочек и кожи и увеличением содержания противовоспалительных цитокинов $(\mathrm{R}=0,49$, $\mathrm{p}<0,05$, по Спирмену). Повышение уровня противовоспалительных цитокинов IL-4, IL-10 и IL-13 при разработанных методах про-

\section{Литература}

1. Oral complications of cancer and cancer therapy: from cancer treatment to survivorship / J. B. Epstein [et al.] // CA Cancer J. Clin. - 2012. - Vol. 62, № 6. - P. 400-422. - doi: 10.3322/ caac. 2115 .

2. Sonis, S. T. Mucositis: The impact, biology and therapeutic opportunities of oral mucositis / S. T. Sonis // Oral Oncol. - 2009. - Vol. 45, № 12. - P. 1015-1020. - doi: 10.1016/j. oraloncology.2009.08.006.

3. MASCC/ISOO clinical practice guidelines for the management of mucositis secondary to cancer therapy / R. V. Lalla [et al.] // Cancer. - 2014. - Vol. 120, № 10. - P. 1453-1461. - doi: 10.1002/cncr.28592.

4. Villa, A. Mucositis: pathobiology and management / A. Villa, S. T. Sonis // Curr Opin Oncol. - 2015. - Vol. 27, № 3. - P. 159-164. - doi: 10.1097/CCO.0000000000000180.

5. Systematic review of laser and other light therapy for the management of oral mucositis in cancer patients / C. Migliorati [et al.] // Support Care Cancer. - 2013. - Vol. 21, № 1. - P. 333-341. - doi: 10.1007/s00520-012-1605-6.

6. Effect of prophylactic low level laser therapy on oral mucositis: a systematic review and meta-analysis / S. Oberoi [et al.] // PLoS One. - 2014. - Vol. 9, № 9. e107418. - doi: 10.1371/journal.pone.0107418.

7. Профилактика и лечение лучевых поражений / Л. И. Мусабаева [и др.] // Онкохирургия. - 2013. - Т. 5, № 2. - C. 76-81.

8. Особенности субпопуляционного состава лимфоцитов периферической крови и инфильтрирующих опухоль у больных плоскоклеточным раком головы и шеи / Т. Н. Заботина [и др.] // Иммунология. - 2019. - Т. 40, № 3. C. 10-19. - doi: 10.24411/0206-4952-2019-13002.

9. Черешнев, В. А. Иммунология воспаления: роль цитокинов / В. А. Черешнев, Е. Ю. Гусев // Медицинская иммунология. - 2001. - Т. 3, № 3. - С. 361-368.

10. Yamaguchi, Y. Overview of current cancer immunotherapy / Y. Yamaguchi // Immunotherapy of Cancer: An Innovative Treatment Comes of Age / ed.: Y. Yamaguchi. - Tokyo, филактики соответствовало меньшей степени лучевых реакций слизистых оболочек и кожи (0-1 степень), при этом в контрольной группе наблюдалось стойкое снижение концентрации данных цитокинов в периферической крови при более выраженной степени лучевых реакций нормальных тканей (2-3 степень).

Таким образом, магнитолазерная терапия, локальная гипоксия и их комплексное применение - эффективные методы ранней медицинской реабилитации пациентов со злокачественными новообразованиями головы и шеи во время лучевой терапии, благодаря которым можно добиться более выраженного противовоспалительного действия, стимуляции репаративных процессов и уменьшить время восстановительного периода. Использование разработанных методов привело к значительным различиям показателей иммунитета в периферической крови пациентов по сравнению с контрольной группой. Полученные данные позволяют утверждать, что иммунокорригирующий эффект этих методов достигается за счет увеличения содержания иммуномедиаторов и поддержания баланса между про- и противовоспалительными цитокинами.

2016. - Ch. 1. - P. 3-17. - doi: 10.1007/978-4-431-5503101.

\section{References}

1. Epstein JB, Thariat J, Bensadoun RJ, Barasch A, Murphy BA, Kolnick L, Popplewell L, Maghami E. Oral complications of cancer and cancer therapy: from cancer treatment to survivorship. CA Cancer $J$ Clin. 2012;62(6):400-22. doi: 10.1016/j.cden.2007.09.004.

2. Sonis ST. Mucositis: The impact, biology and therapeutic opportunities of oral mucositis. Oral Oncol. 2009;45(12):1015-20. doi: 10.1016/j. oraloncology.2009.08.006.

3. Lalla RV, Bowen J, Barasch A, Elting L, Epstein J, Keefe DM, McGuire DB, Migliorati C, NicolatouGalitis O, Peterson DE, Raber-Durlacher JE, Sonis ST, Elad S; Mucositis Guidelines Leadership Group of the Multinational Association of Supportive Care in Cancer and International Society of Oral Oncology (MASCC/ ISOO). MASCC/ISOO clinical practice guidelines for the management of mucositis secondary to cancer therapy. Cancer. 2014;120(10):1453-61. doi: 10.1002/cncr.28592.

4. Villa A, Sonis ST. Mucositis: pathobiology and management. Curr Opin Oncol. 2015;27(3):159-64. doi: 10.1097/ CCO.0000000000000180.

5. Migliorati C, Hewson I, Lalla RV, Antunes HS, Estilo CL, Hodgson B, Lopes NN, Schubert MM, Bowen J, Elad S; Mucositis Study Group of the Multinational Association of Supportive Care in Cancer/International Society of Oral Oncology (MASCC/ISOO). Systematic review of laser and other light therapy for the management of oral mucositis in cancer patients. Support Care Cancer. 2013;21(1):333-41. doi: 10.1007/s00520-012-1605-6.

6. Oberoi S, Zamperlini-Netto G, Beyene J, Treister NS, Sung L. Effect of prophylactic low level laser therapy on oral mucositis: a systematic review and meta-analysis. PLoS One. 2014;9(9):e107418. doi: 10.1371/journal. pone. 0107418 . 
7. Musabaeva LI, Velikaya VV, Gribova OV, Startseva ZhA, Aleinik AN. Profilaktika i lechenie luchevyh porazhenij [Prevention and management of radiation-induced skin injuries]. Onkohirurgija [Oncosurgery]. 2013;5(2):76-81. (Russian).

8. Zabotina TN, Chertkova AI, Tsiklauri VT, Korotkova OV, Zaharova EN, Tabakov DV, Borunova AA, Panchuk IO, Zaderenko IA, Mudunov AM, Kadagidze ZG. Osobennosti subpopuljacionnogo sostava limfocitov perifericheskoj krovi i infiltrirujushhih opuhol u bolnyh ploskokletochnym rakom golovy i shei [Features of peripheral blood lymphocytes and tumor infiltrating lymphocytes subpopulations in patients with squamous cell head and neck cancer]. Immunologiya [Immunology]. 2019;40(3):10-19. doi: 10.24411/0206-4952-2019-13002. (Russian).

9. Chereshnev VA, Gusev EYu. Immunologija vospalenija: rol citokinov [Immunology of inflammation: the role of cytokines]. Meditsinskaya immunologiya [Medical immunology]. 2001;3(3):361-368. (Russian).

10. Yamaguchi Y. Overview of current cancer immunotherapy. In: Yamaguchi Y, editor. Immunotherapy of Cancer. Tokyo: Springer; 2016. Ch. 1; p. 3-17. doi: 10.1007/9784-431-55031-0_1

\title{
CHANGES IN IMMUNITY PARAMETERS DURING RADIOTHERAPY FOR HEAD AND NECK CANCER DEPENDING ON THE METHOD OF RADIATION TOXICITY PREVENTION L. B. Parkhomenko
}

\author{
Belarusian Medical Academy of Post-Graduate Education, Minsk, Belarus
}

Background: The use of magnetolaser and local hypoxia during radiotherapy for head and neck cancer leads to decrease in acute toxicity.

Purpose: To reduce the severity of acute toxicity in patients with head and neck cancer using the developed methods of prevention.

Material and Methods: A prospective randomized study included 120 patients with head and neck cancer. A comparative assessment of the effectiveness of the three developed methods of prevention was carried out in comparison with traditional prevention.

Results: The use of magnetolaser, local hypoxia and their combination led to decrease in the level of pro-inflammatory cytokines and increase in anti-inflammatory cytokines as compared with the control group. This corresponded to a lesser degree of acute toxicity in the main groups.

Conclusions: The use of magnetolaser and/or hypoxia reduces acute toxicity, which is confirmed by changes in immunity parameters.

Keywords: head and neck cancer, radiation toxicity, magnetolaser, local hypoxia, cytokines

For citation: Parkhomenko LB. Changes in immunity during radiotherapy for head and neck cancer depending on the method of radiation toxicity prevention. Journal of the Grodno State Medical University. 2021;19(2):212-218. https://doi.org/10.25298/22218785-2021-19-2-212-218.

Конфликт интересов. Автор заявляет об отсутствии конфликта интересов.

Conflict of interest. The author declare no conflict of interest.

Финансирование. Исследование проведено без спонсорской поддержки.

Financing. The study was performed without external funding.

Соответствие принципам этики. Исследование одобрено локальным этическим комитетом.

Conformity with the principles of ethics. The study was approved by the local ethics committee.

Об авторах / About the authors

Пархоменко Лариса Борисовна / Parkhomenko Larisa, e-mail: 1_vashkevich@tut.by, ORCID: 0000-0002-2832-0124.

Поступила / Received: 20.01.2021

Принята к публикации / Accepted for publication: 18.03.2021 